\title{
a2AP regulates vascular alteration by inhibiting VEGF signaling in systemic sclerosis: the roles of a2AP in vascular dysfunction in systemic sclerosis
}

\author{
Yosuke Kanno $^{1 *}$, En Shu ${ }^{2}$ Hiroyuki Kanoh², Ayaka Matsuda ${ }^{1}$ and Mariko Seishima²
}

\begin{abstract}
Background: Systemic sclerosis (SSC) is a connective tissues disease of unknown origin characterized by vascular damage and extensive fibrosis. Recently, we demonstrated that a2-antiplasmin (a2AP) is associated with the development of fibrosis in SSc. We herein investigate the roles of a2AP in vascular dysfunction in SSc.

Methods: Vascular damage in mice was determined by the levels of blood vessels and blood flow. Vascular functions in vascular endothelial cells (ECS) were determined by the levels of tube formation, cell proliferation, and endothelial junction-associated protein (VE-cadherin and PECAM1) production.

Results: The administration of a2AP induced vascular damage in mice. Conversely, the a2AP neutralization improved vascular damage in a bleomycin-induced mouse model of SSc. Additionally, we showed that the SSC fibroblast-conditioned media induced the reduction of tube formation, cell proliferation, and endothelial junctionassociated protein production in ECs, and that a2AP neutralization improved them. We also examined the mechanisms underlying the effects of a2AP on vascular alteration in SSC and found that a2AP attenuated vascular endothelial growth factor-induced tube formation, cell proliferation, and endothelial junction-associated protein production through the adipose triglyceride lipase/tyrosine phosphatase SHP2 axis in ECs.

Conclusion: Our findings demonstrate that a2AP is associated with vascular alteration, and that the blocking of a2AP improves vascular dysfunction in SSC.
\end{abstract}

Keywords: Alpha2-antiplasmin, Systemic sclerosis, Vascular dysfunction, VEGF

\section{Background}

Systemic sclerosis (SSc) is a connective tissue disease characterized by vascular damage and fibrosis of skin and visceral organs [1]. Vascular damage, such as the reduction of blood vessels and blood flow, occurs in the early stages of the disease, and leads to extensive fibrosis [2]. However, the detailed mechanisms of SSc pathogenesis is unclear. Vascular endothelial growth factor (VEGF) is known to regulate the growth and activation of vascular endothelial cells (ECs), and plays a critical

\footnotetext{
* Correspondence: ykanno@dwc.doshisha.ac.jp

${ }^{1}$ Department of Clinical Pathological Biochemistry, Faculty of Pharmaceutical Science, Doshisha Women's Collage of Liberal Arts, 97-1 Kodo, Kyo-tanabe, Kyoto 610-0395, Japan

Full list of author information is available at the end of the article
}

role in maintaining the vascular function. The expression of VEGF is elevated in various cells, such as fibroblasts, ECs, and immune cells, but vascular insufficiency manifests in SSc $[2,3]$. The impairment of VEGF responses may cause vascular dysfunction in SSc. However, the detailed mechanisms are still not precisely understood.

Alpha2-antiplasmin ( $\alpha 2 \mathrm{AP})$ functions as the main inhibitor of plasmin, resulting in the formation of a stable inactive complex, plasmin- $\alpha 2 \mathrm{AP}$ and inhibits fibrinolysis [4]. $\alpha 2 \mathrm{AP}$ is known to be synthesized in various tissues [5]. Recently, we found that $\alpha 2 \mathrm{AP}$ induces TGF- $\beta$ production through adipose triglyceride lipase (ATGL), which has been described as a member of the calciumindependent phospholipase $\mathrm{A}_{2}$ /adiponutrin/patatin-like 
phospholipase domain-containing 2 (PNPLA2) family, and has a pro-fibrotic effects other than regulation of plasmin activity [6-10]. We also found that the expression of $\alpha 2 \mathrm{AP}$ was elevated in the dermal fibroblasts obtained from SSc patients and the fibrotic tissue in SSc mouse models, and $\alpha 2 \mathrm{AP}$ is associated with the development of fibrosis in SSc [7, 10]. Additionally, $\alpha 2 \mathrm{AP}$ is known to play a critical role on angiogenesis, tissue repair, and vascular remodeling $[11,12]$, and may be also associated with vascular alteration in SSc. We herein investigated that the roles of $\alpha 2 \mathrm{AP}$ in vascular dysfunction in SSc.

\section{Methods}

\section{Mice experiments}

We performed mice experiments as previously described [10]. The saline, bleomycin $(5 \mathrm{mg} / \mathrm{kg}$ ) plus control IgG $(100 \mu \mathrm{g} / \mathrm{kg})$ or bleomycin $(5 \mathrm{mg} / \mathrm{kg})$ plus anti- $\alpha 2 \mathrm{AP}$ antibodies $(100 \mu \mathrm{g} / \mathrm{kg})(\mathrm{R} \& \mathrm{D}$ Systems, MN, USA) were administered subcutaneously into the shaved backs of mice (male, 8-week-old C57BL/6 J mice) in the same site daily for up to 3 weeks. In parallel experiments, the saline or $\alpha 2 \mathrm{AP}(15 \mu \mathrm{g} / \mathrm{kg})$ (Calbiochem, CA, USA) were administered subcutaneously into the shaved backs of mice (male, 8-week-old C57BL/6 J mice) in the same site daily for up to 3 weeks. The samples of skin were placed immediately in liquid nitrogen, and stored at $-80{ }^{\circ} \mathrm{C}$ until further use.

\section{Immunohistochemical staining of PECAM1}

We performed immunohistochemical staining as previously described $[10,11]$. Paraffin sections were labeled with anti-PECAM1 antibody, then secondarily labeled with FITC-conjugated anti-rabbit IgG (Thermo Scientific, CA, USA). We used Rabbit (DA1E) mAb IgG XP Isotype control (Cell Signaling Technology, MA, USA) as isotype control (Additional file 1: Figure S1). The signals in the skin section were detected using a laserscanning microscope. Then, the signals obtained from the same rectangular area for the dermis in the skin section were analyzed using ImageJ.

\section{Blood flow in the skin}

Blood flow in the skin was measured for 10 seconds using a laser Doppler flowmeter (BRL-100; Bio Research Center, Tokyo, Japan), and determined by calculating the average of two-time measurements in each skin sample.

\section{Cell culture}

Human normal and SSc dermal fibroblasts were obtained from patients with SSc (S4) and healthy control (N3) as previously described [10, 11]. Dermal fibroblasts were seeded onto the $10-\mathrm{cm}$ diameter dishes and maintained in $10 \mathrm{~mL}$ Dulbecco's modified Eagle medium
(DMEM) containing $10 \%$ FCS at $37{ }^{\circ} \mathrm{C}$ in a humidified atmosphere with $5 \% \mathrm{CO}_{2} / 95 \%$ air. After 5 days, the media were collected. In other studies, vascular ECs (UV $\bigcirc 2$ ) were seeded onto $35-\mathrm{mm}$ diameter dishes and maintained in $2 \mathrm{~mL}$ DMEM containing $10 \% \mathrm{FCS}$ at $37^{\circ}$ $\mathrm{C}$ in a humidified atmosphere with $5 \% \mathrm{CO}_{2} / 95 \%$ air. After 5 days, the media were replaced with serum-free DMEM. Then, the cells were used for experiments.

\section{Tube formation assay}

Matrigel (Becton, Dickinson and Company, NJ, USA) was added to each well of a 96-well plate. ECs were seeded on Matrigel coated plates, and were treated with the conditioned media (CM) of dermal fibroblasts, VEGF, or $\alpha 2 \mathrm{AP}$ at the indicated concentration for 24 hours. The length of capillary like structure was analyzed by using ImageJ.

\section{Cell proliferation assays}

ECs were seeded on a 96-well plate, and the ECs were treated with the $\mathrm{CM}$ of dermal fibroblasts, VEGF, or $\alpha 2 \mathrm{AP}$ at the indicated concentration for 24 hours. Cell proliferation was determined by counting cells number.

\section{Western blot analysis}

Cells were washed twice with cold PBS, harvested, and then sonicated in lysis buffer containing $10 \mathrm{mM}$ Tris$\mathrm{HCl}$ buffer (pH 7.5), 1\% SDS, $1 \%$ Triton X-100, and a protease inhibitor cocktail (Roche, Mannheim, Germany). The skin samples from mice were homogenized and sonicated in the lysis buffer. The protein concentration in each lysate was measured using a BCA protein assay kit (Pierce, IL, USA). Proteins in the supernatant were separated by electrophoresis on 10\% SDSpolyacrylamide gels and transferred to a PVDF membrane. We detected PECAM1, vascular endothelial cadherin, GAPDH, phospho-VEGFR2, VEGFR2, phosphoAkt, Akt, phospho-ERK1/2, ERK1/2, phospho-p38, p38, phospho-SHP2, SHP2, and ATGL by incubation with the respective antibodies followed by incubation with horseradish peroxidase-conjugated antibodies to rabbit IgG (Amersham Pharmacia Biotech, Uppsala, Sweden).

\section{ATGL siRNAs study}

SSc dermal fibroblasts were transfected with ATGL siRNA (Santa Cruz Biotechnology, CA, USA) using Lipofectamine 2000 (Invitrogen, CA, USA) according to the manufacturer's instructions. A non-specific siRNA was employed as the control. At 24 hours after transfection, the cells were used for experiments.

\section{Statistical analysis}

All data were expressed as mean $\pm \mathrm{SEM}$. The significance of the effect of each treatment $(P<0.05)$ was 
determined by analysis of variance (ANOVA) followed by the least significant difference test.

\section{Results}

Effect of a2AP on vascular damage in mice

To clarify the effects of $\alpha 2 \mathrm{AP}$ on vascular damage, such as the reduction of blood vessels and blood flow, we examined the expression of the vascular EC marker, platelet-endothelial cell adhesion molecule 1 (PECAM1, CD31) and blood flow following the administration of $\alpha 2 A P$ in mice. The administration of $\alpha 2 A P$ induced the reduction of PECAM1 expression within the dermis (Fig. 1a, b) and blood flow in the skin (Fig. 1c).

\section{Effect of blocking a2AP on vascular damage in a bleomycin-induced mouse model of SSc}

We examined the effects of $\alpha 2 \mathrm{AP}$ neutralization on vascular damage in a bleomycin-induced mouse model of SSc. The administration of bleomycin induced the reduction of PECAM1 expression (Fig. 2a, b) and blood flow (Fig. 2c) in the skin of SSc model mice, and the blocking of $\alpha 2 \mathrm{AP}$ by $\alpha 2 \mathrm{AP}$-neutralizing antibodies improved the bleomycin-induced reduction of PECAM1 expression within the dermis (Fig. 2a, b) and blood flow (Fig. 2c) in the skin of SSc model mice.

\section{Effect of blocking a2AP on the SSc dermal fibroblast-} induced vascular dysfunction in ECs

We examined whether or not the CM from SSc dermal fibroblasts induces vascular dysfunction, including the reduction of tube formation, cell proliferation, and endothelial junction-associated protein (PECAM1 and vascular endothelial cadherin) production. The tube formation (Fig. 3a, b), cell proliferation (Fig. 3c), and endothelial junction-associated protein production (Fig. 3d) in SSc dermal fibroblast CM-stimulated ECs were lower than those in normal dermal fibroblast CM-stimulated ECs. These data suggest the SSc dermal fibroblasts induced vascular dysfunction. Next, we examined the effects of $\alpha 2 \mathrm{AP}$ neutralization on the SSc dermal fibroblast CM-induced reduction of tube formation, cell proliferation, and endothelial junction-associated protein production. The $\alpha 2 \mathrm{AP}$ neutralization improved the SSc dermal fibroblast CM-induced reduction of tube formation (Fig. 3e, f), cell proliferation (Fig. 3g), and endothelial junction-associated protein production (Fig. 3h).

\section{Effect of a2AP on the VEGF-induced pro-angiogenic ef-} fects in ECs

We examined the effects of $\alpha 2 A P$ on the VEGFregulated tube formation, cell proliferation, and endothelial junction-associated protein production in ECs. We confirmed that VEGF induced tube formation (Fig. 4a, b), cell proliferation (Fig. 4c), and endothelial junction-associated protein production (Fig. 4d) in ECs, and found that $\alpha 2 \mathrm{AP}$ attenuated VEGF-induced tube formation (Fig. 4a, b), cell proliferation (Fig. 4c), and endothelial junction-associated protein production (Fig. 4d) in ECs.

\section{a}

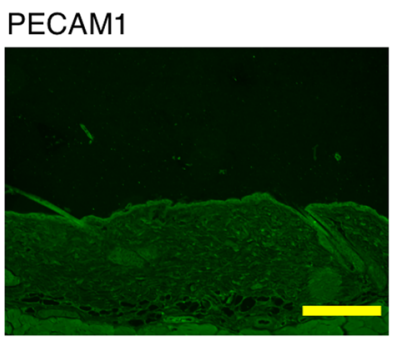

Saline

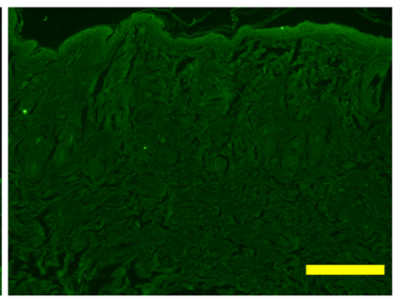

$\alpha 2 A P$

\section{b}

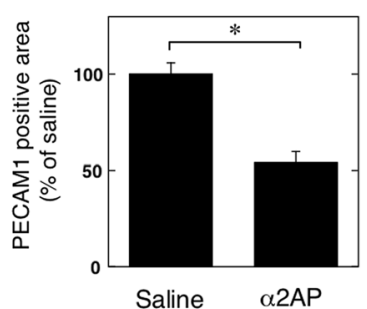

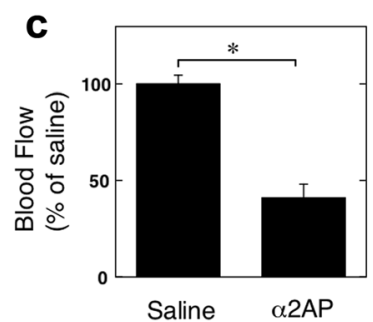

Fig. 1 Effect of a2AP on vascular damage in mice. a The skin sections from saline or a2AP-administered mice were stained with antibodies to PECAM1. b The histogram shows quantitative representations of PECAM1 $(n=6)$. c Blood flow in the skin of saline or a2AP-administered mice $(n=4)$. The data represent the mean \pm SEM. ${ }^{*} P<0.01$. Scale bar, $200 \mu \mathrm{m}$ 
a

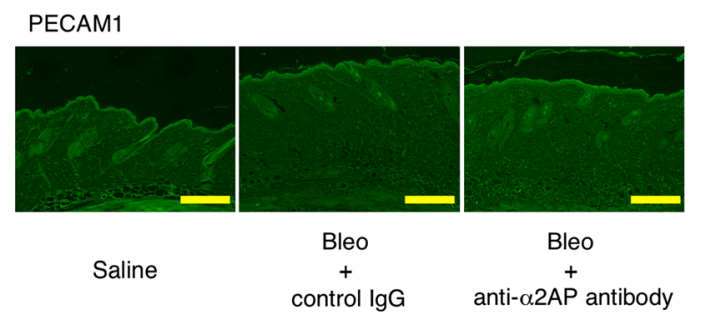

b

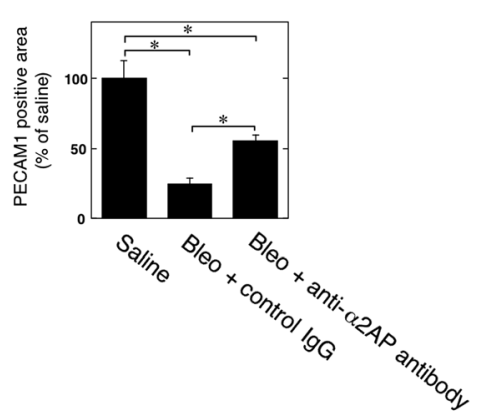

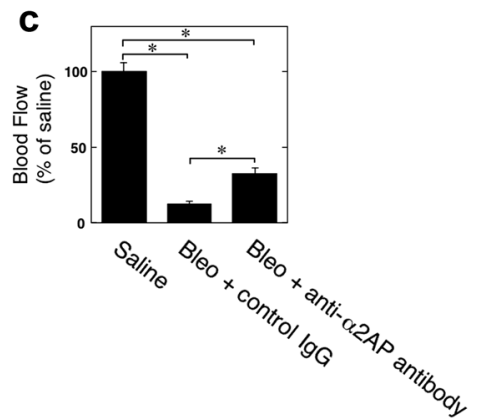

Fig. 2 Effect of blocking a2AP on vascular damage in a bleomycin-induced mouse model of systemic sclerosis. a The skin sections from mice treated with saline, bleomycin plus control lgG, or bleomycin plus a2AP-neutralizing antibodies were stained with antibodies to PECAM1. b The histogram shows quantitative representations of PECAM1 $(n=6)$. c Blood flow in the skin of mice treated with saline $(n=5)$, bleomycin plus control $\lg G(n=4)$, or bleomycin plus a2AP-neutralizing antibodies $(n=4)$. The data represent the mean \pm SEM. ${ }^{*} P<0.01$. Scale bar, $200 \mu \mathrm{m}$

\section{Effect of a2AP on VEGF signaling through ATGL/SHP2}

\section{axis in ECs}

We also examined the effects of $\alpha 2 A P$ on VEGF signaling in ECs, and found that $\alpha 2 \mathrm{AP}$ inhibited VEGFinduced VEGFR2, Akt, ERK1/2, and p38 phosphorylation in ECs (Fig. 5a). It has been reported that srchomology domain-2 containing tyrosine phosphatase 2 (SHP2) activation inhibits VEGF signaling [13]. Therefore, we examined whether or not $\alpha 2 \mathrm{AP}$-inhibited VEGF signaling is associated with SHP2 activation in ECs. $\alpha 2 \mathrm{AP}$ induced SHP2 phosphorylation (Fig. $5 \mathrm{~b}$ ) and the SHP2 inhibitor, NSC87877, abrogated $\alpha 2$ AP-inhibited Akt, ERK1/2, and p38 phosphorylation induced by VEGF (Fig. 5c). We then examined whether or not the $\alpha 2 \mathrm{AP}$-induced SHP2 activation is associated with the $\alpha 2 \mathrm{AP}$ receptor, ATGL, in ECs. The reduction of ATGL using siRNA attenuated the $\alpha 2 \mathrm{AP}$-induced SHP2 phosphorylation in ECs (Fig. 5d). We also examined the effects of the irreversible ATGL inhibitor, bromoenol lactone (BEL), on $\alpha 2 \mathrm{AP}$-activated SHP2 in ECs. BEL attenuated $\alpha 2 \mathrm{AP}$-induced SHP2 phosphorylation in ECs (Fig. 5e). Additionally, BEL abrogated $\alpha 2 \mathrm{AP}$-inhibited Akt, ERK1/2, and p38 phosphorylation induced by VEGF in ECs (Fig. 5f).

\section{Discussion}

SSc is a chronic immune disorder characterized by vascular dysfunction and fibrosis of the skin and internal organs [1]. Recently, we showed that $\alpha 2 \mathrm{AP}$ is associated with the development of fibrosis in SSc $[6-8,10] . \alpha 2 \mathrm{AP}$ is also associated with angiogenesis [11], vascular remodeling [12], the production of IgG, IgM, and IgE [14, $15]$, and the recruitment of lymphocytes and neutrophils [15-17]. These observations suggest that $\alpha 2$ AP may be a critical regulator in the pathogenesis of SSc. We herein demonstrated that $\alpha 2 \mathrm{AP}$ is associated with vascular dysfunction in SSc.

We showed that the administration of $\alpha 2 \mathrm{AP}$ induced vascular damage such as the reduction of blood vessels and blood flow in mice (Fig. 1). Conversely, $\alpha 2 \mathrm{AP}$ neutralization improved vascular damage in a bleomycin-induced mouse model of SSc (Fig. 2). These data suggest that $\alpha 2 \mathrm{AP}$ may be one of the factors initiating vascular damage in SSc.

In SSc, fibroblasts are likely to be important effector cells, and SSc fibroblasts inhibit angiogenesis [18, 19]. We therefore examined whether or not SSc fibroblasts induce vascular dysfunction, such as the reduction of tube formation, cell proliferation, and endothelial junction-associated protein production, using CM from human normal and SSc dermal fibroblasts. We found that SSc dermal fibroblasts induced vascular dysfunction (Fig. 3a-d). We also showed that the blocking of $\alpha 2 \mathrm{AP}$ markedly improved SSc dermal fibroblast-induced vascular dysfunction (Fig. 3e-h). In a previous study, we showed that the expression of $\alpha 2 \mathrm{AP}$ was elevated in SSc dermal fibroblasts [10]. The SSc fibroblast-derived $\alpha 2 \mathrm{AP}$ may cause vascular dysfunction in the disease. 
a

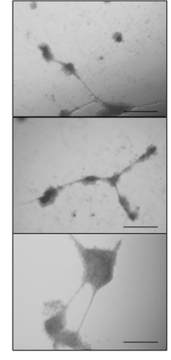

DMEM

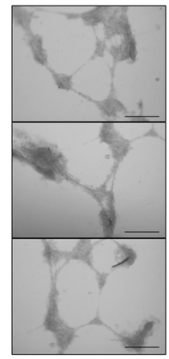

Normal fibroblasts SSc fibroblasts $\mathrm{CM} \quad \mathrm{CM}$

C

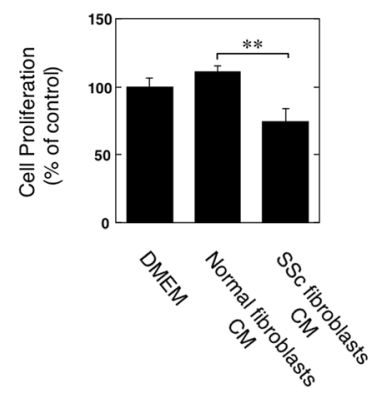

e

SSc fibroblasts $\mathrm{CM}$
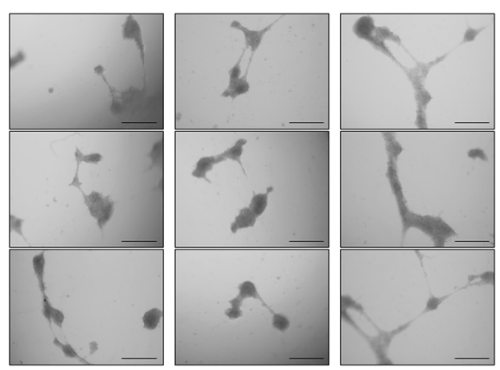

Control lgG Anti- $\alpha$ AP antibody

g

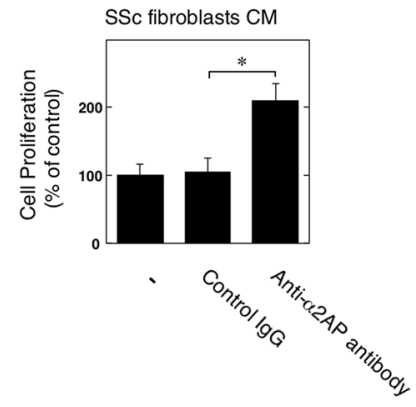

b

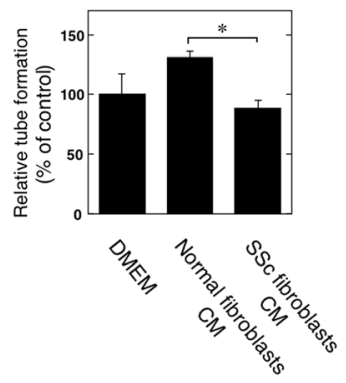

d
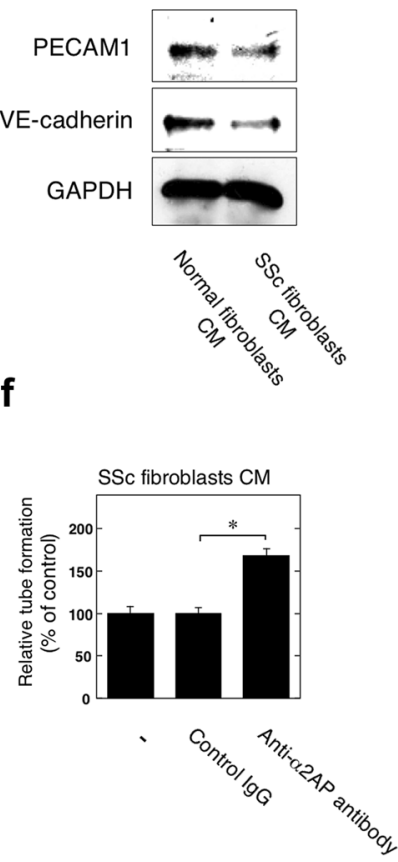

$\mathbf{h}_{\text {SSc fibroblasts } \mathrm{CM}}$

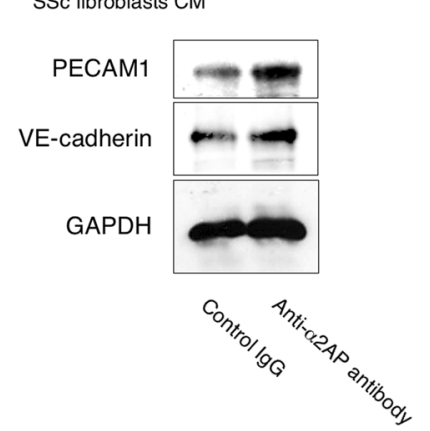

Fig. 3 (See legend on next page.) 
(See figure on previous page.)

Fig. 3 Effect of blocking a2AP on the systemic sclerosis (SSC) dermal fibroblast-induced vascular dysfunction in endothelial cells (ECS). a ECs were seeded on 96-well Matrigel coated plates. ECs were cultured with the condition media (CM) of human normal dermal fibroblasts or human SSC dermal fibroblasts for 24 hours. b Tube formation in ECs was measured as described in Materials and Methods $(n=6)$. c ECs were cultured with the CM of the human normal dermal fibroblasts or the human SSc dermal fibroblasts for 24 hours. The cell proliferation was assessed as described in Materials and Methods $(n=3)$. $\mathbf{d}$ ECs were cultured with the CM of human normal dermal fibroblasts or human SSC dermal fibroblasts for 24 hours. The expression of each protein was examined by western blot analysis. e ECs were seeded on 96-well Matrigel-coated plates. ECs were cultured with the CM of human SSc dermal fibroblasts, and were stimulated by control $\operatorname{lgG}(1 \mu \mathrm{g} / \mathrm{mL})$ or a2AP-neutralizing antibodies (1 $\mu \mathrm{g} / \mathrm{mL})$ for 24 hours. $\mathbf{f}$ Tube formation in ECs was measured as described in Materials and Methods $(n=6)$. $\mathbf{g}$ ECs were cultured with the CM of the human SSc dermal fibroblasts, and were stimulated by control $\operatorname{lgG}(1 \mu \mathrm{g} / \mathrm{mL})$ or a2AP-neutralizing antibodies $(1 \mu \mathrm{g} / \mathrm{mL})$ for 24 hours. Cell proliferation was assessed as described in Materials and Methods $(n=8)$. $\mathbf{h}$ ECs were cultured with the CM of human SSc dermal fibroblasts and were stimulated by control lgG $(1 \mu \mathrm{g} / \mathrm{mL})$ or a2AP-neutralizing antibodies $(1 \mu \mathrm{g} / \mathrm{mL})$ for 24 hours. The expression of each protein was examined by western blot analysis. Data represent the mean \pm SEM. ${ }^{*} P<0.01,{ }^{* *} P<0.05$. Scale bar, $50 \mu \mathrm{m}$

It has been reported that the expression of VEGF, which is a main regulator of angiogenesis, is elevated in SSc patients $[2,3]$. However, angiogenesis is disturbed in SSc, and the mechanism of dysregulated angiogenesis in the presence of elevated VEGF remains poorly understood. We showed that $\alpha 2 \mathrm{AP}$ attenuated VEGF-induced proangiogenic effects such as tube formation, cell proliferation, and endothelial junction-associated protein production in ECs (Fig. 4). Additionally, we showed that $\alpha 2 \mathrm{AP}$ inhibited VEGF signaling (VEGFR2, Akt, ERK1/2, and p38 activation) (Fig. 5a). It has been reported that the activation of SHP2 inhibits VEGF signaling and regulates vascular endothelial functions [13]. In this study, we found that a2AP induced SHP2 activation (Fig. 5b), and the inhibition of SHP2 recovered $\alpha 2 A P$-attenuated VEGF signaling (Fig. 5c). We also found that $\alpha 2 A P$ inhibited VEGF signaling through SHP2 activation. We previously showed that $\alpha 2 \mathrm{AP}$ induces cell differentiation and TGF- $\beta$ production through ATGL [8]. Therefore, we examined whether or not ATGL is associated with $\alpha 2 \mathrm{AP}$-induced SHP2 activation using siRNA and its inhibitor. Both reduction and inhibition of ATGL attenuated the $\alpha 2$ AP-induced SHP2 activation (Fig. 5d, e). Additionally, the inhibition of ATGL recovered the $\alpha 2 \mathrm{AP}$-inhibited VEGF signaling (Fig. 5f). These data suggest that $\alpha 2 \mathrm{AP}$ induced SHP2 activation through ATGL, and the $\alpha 2 \mathrm{AP}$-activated SHP2 inhibited VEGF signaling (Fig. 5g). The increase of $\alpha 2 A P$ expression in SSc may cause impairment of the VEGF response, and lead to vascular dysfunction.

Additionally, plasmin is known to regulate vascular endothelial functions, and influence the progression of various cardiovascular diseases through fibrinolysis, the degradation of matrix proteins, and the activation of growth factors [20]. The levels of plasmin- $\alpha 2 A P$ and Ddimer are elevated in patients with SSc [21, 22], and plasmin may also affect vascular dysfunction in SSc. $\alpha 2 \mathrm{AP}$ may cause vascular disorder not only through inhibition of VEGF responses but also through plasmin inhibition.
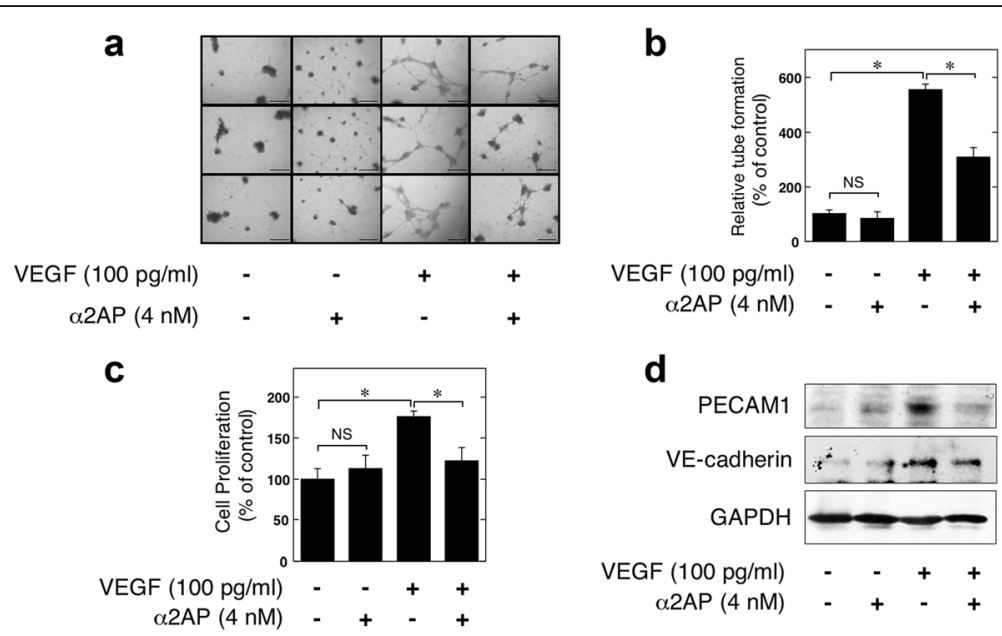

Fig. 4 Effect of a2AP on the VEGF-induced pro-angiogenic effects in endothelial cells (ECs). a ECs were seeded on 96-well Matrigel-coated plates. ECs were cultured in the absence or presence of VEGF $(100 \mathrm{pg} / \mathrm{mL})$ or a2AP $(4 \mathrm{nM})$ as indicated for 24 hours. $\mathbf{b}$ Tube formation in ECs was measured as described in Materials and Methods $(n=6)$. c ECs were cultured for 24 hours in the absence or presence of VEGF $(100 \mathrm{pg} / \mathrm{mL})$ or a2AP (4 nM). Cell proliferation was assessed as described in Materials and Methods $(n=3)$. $\mathbf{d}$ ECs were cultured for 24 hours in the absence or presence of VEGF $(100 \mathrm{pg} / \mathrm{mL})$ or a2AP $(4 \mathrm{nM})$. The expression of each protein was examined by western blot analysis. The data represent the mean \pm SEM. ${ }^{*} P<0.01$. NS, not significant. Scale bar, $50 \mu \mathrm{m}$ 


\section{a}

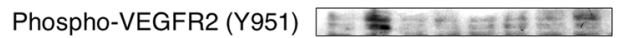

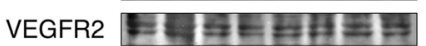

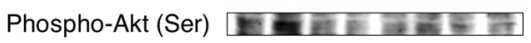

Akt

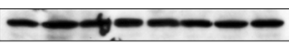

Phospho-ERK $1 / 2$ =

ERK $1 / 2$

Phospho-p38

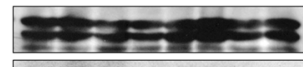

p38

$\operatorname{VEGF}(100 \mathrm{pg} / \mathrm{ml}) \quad 01530600153060$ (min)

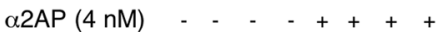

C

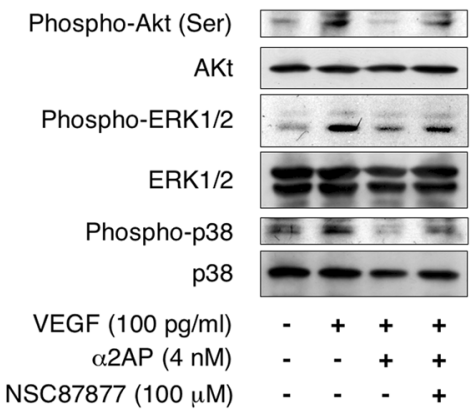

b

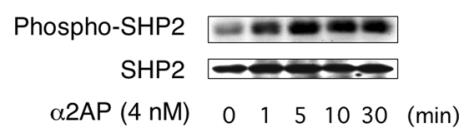

b

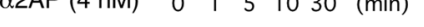

d

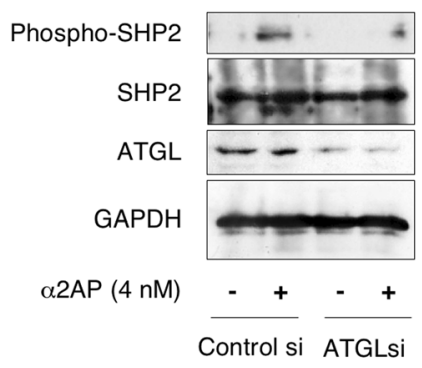

e

g

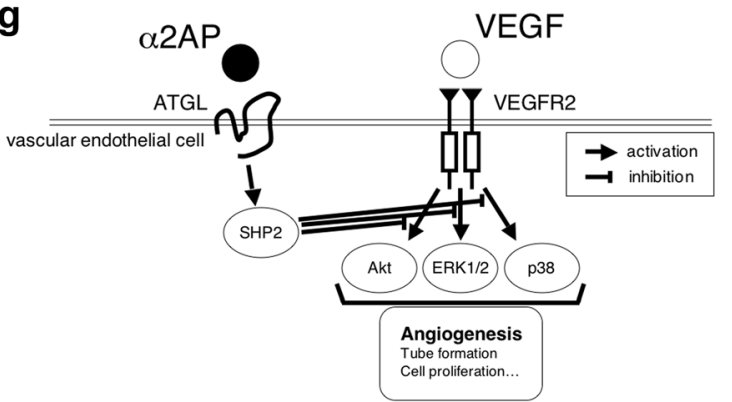

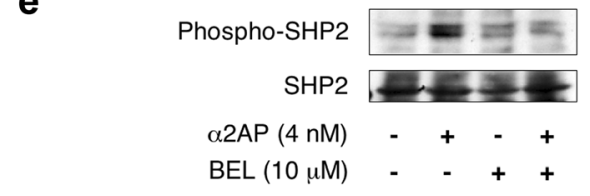

Fig. 5 Effect of a2AP on the VEGF signaling through ATGL/SHP2 axis in endothelial cells (ECs). a ECs were pretreated with 4 nM a2AP for 30 minutes and then stimulated with $100 \mathrm{pg} / \mathrm{mL}$ VEGF for the indicated periods. Phosphorylation of each protein was examined by western blot analysis. b ECs were stimulated with $4 \mathrm{nM}$ a2AP for the indicated periods. Phosphorylation of SHP2 was examined by western blot analysis. $\mathbf{c}$ ECs were cultured for 30 minutes in the absence or presence of $4 \mathrm{nM}$ a2AP or $100 \mu \mathrm{M}$ NSC87877, and then stimulated with $100 \mathrm{pg} / \mathrm{mL}$ VEGF for 15 minutes. Phosphorylation of each protein was examined by western blot analysis. $\mathbf{d}$ ECs were transfected with control or ATGL siRNA. At 24 hours after transfection, the cells were stimulated with $4 \mathrm{nM}$ a2AP for 5 minutes. The expression of each protein was examined by western blot analysis. e ECs were pretreated with $10 \mu \mathrm{M}$ BEL for 30 minutes and then stimulated with $4 \mathrm{nM}$ a2AP for 5 minutes. Phosphorylation of SHP2 was examined by western blot analysis. f ECs were pretreated with $4 \mathrm{nM}$ a2AP or $10 \mu \mathrm{M}$ BEL for 30 minutes and then stimulated with $100 \mathrm{pg} / \mathrm{mL}$ VEGF for 15 minutes. Phosphorylation of each protein was examined by western blot analysis. $\mathbf{g}$ The proposed mechanism of a2AP-attenuated vascular endothelial functions. VEGF induced Akt, ERK1/2, and p38 activation and led to pro-angiogenic effects, such as tube formation, cell proliferation, and endothelial junction-associated protein production in ECs. On the other hand, a2AP activated SHP2 through ATGL, and then a2AP-induced SHP2 activation inhibited VEGF signaling in ECs. a2AP inhibited VEGF signaling through the ATGL/SHP2 axis, and may cause impairment of vascular functions 


\section{Conclusion}

$\alpha 2 \mathrm{AP}$ functions as an inducer of vascular damage in mice. Blocking of $\alpha 2 \mathrm{AP}$ improved vascular damage in an SSc mice model and SSc dermal fibroblast-induced vascular dysfunction. Additionally, $\alpha 2 \mathrm{AP}$ regulated vascular alteration by inhibiting VEGF signaling through the ATGL/SHP2 axis. Our findings may eventually provide new insights into the development of clinical therapies for SSc.

\section{Additional file}

Additional file 1: Figure S1. (A) The skin sections from saline or a2APadministered mice were stained with isotype control. (B) The skin sections from mice treated with saline, bleomycin plus control lgG, or bleomycin plus a2AP-neutralizing antibodies were stained with isotype control. Scale bar, $200 \mu \mathrm{m}$. (TIF $1805 \mathrm{~kb}$ )

\section{Abbreviations}

ATGL: adipose triglyceride lipase; BEL: bromoenol lactone; CM: conditioned media; EC: endothelial cell; PECAM1: platelet-endothelial cell adhesion molecule 1; PNPLA2: patatin-like phospholipase domain-contain 2; SHP2: src-homology domain-2 containing tyrosine phosphatase 2; SSc: systemic sclerosis; VEGF: vascular endothelial growth factor; a2AP: a2-antiplasmin

\section{Acknowledgements}

We thank the support of the Takeda Science Foundation.

\section{Funding}

This work was supported by the Takeda Science Foundation.

\section{Availability of data and materials}

The authors declare that all data supporting the findings of this study are available within the article.

\section{Authors' contributions}

YK conceived and designed the experiment, and was involved in human and mice experiments, analyzed the data, data interpretation, and writing of the manuscript. ES was involved in human experiments, data interpretation, and writing of the manuscript. HK was involved in human experiments, data interpretation, and writing of the manuscript. AM was involved in mice experiments and analyzed the data. MS was involved in human experiments, data interpretation, and writing of the manuscript. All authors read and approved the final manuscript.

\section{Competing interests}

The authors declare that they have no competing interests.

\section{Consent for publication}

All authors approved the manuscript for submission.

\section{Ethics approval and consent to participate}

The animal experiments in this study were approved by the Animal Research Committee of Doshisha Women's College of Liberal Arts (Approval ID: Y15-026). The human experiments in this study were approved by Doshisha Women's College of Liberal Arts Research Ethics Committee and Gifu University Graduate School of Medicine Ethics Committee (Approved ID: 39, and 26-112, respectively). We received written, informed patient consent.

\section{Author details}

'Department of Clinical Pathological Biochemistry, Faculty of Pharmaceutical Science, Doshisha Women's Collage of Liberal Arts, 97-1 Kodo, Kyo-tanabe, Kyoto 610-0395, Japan. ²Department of Dermatology, Gifu University Graduate School of Medicine, 1-1 Yanagido, Gifu 501-1194, Japan.

\section{References}

1. Desbois AC, Cacoub P. Systemic sclerosis: an update in 2016. Autoimmun Rev. 2016;15:417-26

2. Liakouli V, Cipriani P, Marrelli A, Alvaro S, Ruscitti P, Giacomelli R. Angiogenic cytokines and growth factors in systemic sclerosis. Autoimmun Rev. 2011;10: 590-4.

3. Trojanowska M. Cellular and molecular aspects of vascular dysfunction in systemic sclerosis. Nat Rev Rheumatol. 2010;6:453-60.

4. Collen D. Identification and some properties of a new fast-reacting plasmin inhibitor in human plasma. Eur J Biochem. 1976;69:209-16.

5. Menoud PA, Sappino N, Boudal-Khoshbeen M, Vassalli JD, Sappino AP. The kidney is a major site of alpha(2)-antiplasmin production. J Clin Invest. 1996; 97:2478-84.

6. Kanno Y, Kuroki A, Okada K, Tomogane K, Ueshima S, Matsuo O, et al. alpha2-Antiplasmin is involved in the production of transforming growth factor beta1 and fibrosis. J Thromb Haemost. 2007;5:2266-73.

7. Kanno Y, Kawashita E, Minamida M, Kaneiwa A, Okada K, Ueshima S, et al. alpha2-antiplasmin is associated with the progression of fibrosis. Am J Pathol. 2010;176:238-45.

8. Kanno Y, Kawashita E, Kokado A, Okada K, Ueshima S, Matsuo O, et al. Alpha2-antiplasmin regulates the development of dermal fibrosis in mice by prostaglandin $\mathrm{F}(2 \mathrm{a})$ synthesis through adipose triglyceride lipase/calciumindependent phospholipase A(2). Arthritis Rheum. 2013;65:492-502.

9. Kanno Y, Kawashita E, Kokado A, Kuretake H, Ikeda K, Okada K, et al. a2AP mediated myofibroblast formation and the development of renal fibrosis in unilateral ureteral obstruction. Sci Rep. 2014:4:5967.

10. Kanno Y, Shu E, Kanoh H, Seishima M. The antifibrotic effect of a2AP neutralization in systemic sclerosis dermal fibroblasts and mouse models of systemic sclerosis. J Invest Dermatol. 2016;136:762-9.

11. Kanno Y, Hirade K, Ishisaki A, Nakajima K, Suga H, Into T, et al. Lack of alpha2-antiplasmin improves cutaneous wound healing via over-released vascular endothelial growth factor-induced angiogenesis in wound lesions. J Thromb Haemost. 2006:4:1602-10.

12. Hou Y, Okada K, Okamoto C, Ueshima S, Matsuo O. Alpha2-antiplasmin is a critical regulator of angiotensin II-mediated vascular remodeling. Arterioscler Thromb Vasc Biol. 2008;28:1257-62

13. Fraineau S, Monvoisin A, Clarhaut J, Talbot J, Simonneau C, Kanthou C, et al The vitamin K-dependent anticoagulant factor, protein $\mathrm{S}$, inhibits multiple VEGF-A-induced angiogenesis events in a Mer- and SHP2-dependent manner. Blood. 2012;120:5073-83.

14. Zhabin SG, Gorin VS. The effects of alpha 2-antiplasmin complex and alpha 2-antiplasmin on the secretion of lgG and lgM by cultured human mononuclear cells. J Clin Lab Immunol. 1997:49:77-82.

15. Okada K, Ueshima S, Kawao N, Yano M, Tamura Y, Tanaka M, et al. Lack of both a2-antiplasmin and plasminogen activator inhibitor type-1 induces high lgE production. Life Sci. 2013;93:89-95.

16. Kager LM, Weehuizen TA, Wiersinga WJ, Roelofs JJ, Meijers JC, Dondorp AM, et al. Endogenous a2-antiplasmin is protective during severe gram-negative sepsis (melioidosis). Am J Respir Crit Care Med. 2013;188:967-75.

17. Eddy JL, Schroeder JA, Zimbler DL, Bellows LE, Lathem WW. Impact of the Pla protease substrate a2-antiplasmin on the progression of primary pneumonic plague. Infect Immun. 2015;83:4837-47.

18. Gilbane AJ, Denton CP, Holmes AM. Scleroderma pathogenesis: a pivotal role for fibroblasts as effector cells. Arthritis Res Ther. 2013;15:215.

19. Serrati S, Cinelli M, Margheri F, Guiducci S, Del Rosso A, Pucci M, et al. Systemic sclerosis fibroblasts inhibit in vitro angiogenesis by MMP-12dependent cleavage of the endothelial cell urokinase receptor. J Pathol. 2006;210:240-8

20. Plow EF, Hoover-Plow J. The functions of plasminogen in cardiovascular disease. Trends Cardiovasc Med. 2004;14:180-6.

21. Jinnin $\mathrm{M}$, Ihn H, Yamane K, Asano Y, Yazawa N, Tamaki K. Plasma plasminalpha2-plasmin inhibitor complex levels are increased in systemic sclerosis patients with pulmonary hypertension. Rheumatology (Oxford). 2003;42: 240-3.

22. Marie I, Borg JY, Hellot MF, Levesque H. Plasma D-dimer concentration in patients with systemic sclerosis. Br J Dermatol. 2008:158:392-5. 